\title{
Wolf-Rayet stars as a diagnostic of internal mixing processes in massive mass losing stars
}

\author{
N. Langer \\ Universitäts-Sternwarte Göttingen, Geismarlandstraße 11, \\ D-3400 Göttingen, F.R.G.
}

Massive stars $\left(M_{Z A M S} \gtrsim 30 M_{\odot}\right)$ develop during their observable hydrostatic evolutionary phases - i.e. central H- and He-burning - three different large scale convective zones, which are: 1) The $\mathrm{H}$-burning convective core, 2) the intermediate convective shell (ICZ) above the hydrogen shell source, which forms at time of hydrogen exhaustion, and 3 ) the He-burning convective core. The spatial extent of these convective regions, wherein the chemical structure is rapidly homogenised, can be predicted from theory only with a large uncertainty. Different assumptions on the efficiency of these mixing processes in stellar evolution calculations lead to quite different evolutionary pictures for massive stars, especially regarding their Wolf-Rayet (WR) phases. On the other side, many observational data concerning WR stars became available in recent years. For this reason, we attempt to perform a comparison of theoretical evolutionary sequences with observed properties of WR stars in order to derive restrictions on the efficiency of the three mixing processes mentioned above.

1) Effects of greatly enlarged H-burning convective cores are (cf. Langer and El Eid, 1986; Prantzos et al., 1986):

- A reduced width of the main sequence band and the avoidance of the domain of the Luminous Blue Variables (LBVs) in the HR diagram.

- The formation of very massive (luminous) WR stars of types WNE and WC.

Both points disagree with observations: The observed main sequence width requires only a moderate core mass increase (cf. Mermilliod and Maeder, 1986), the LBVs exist, and very massive WNE and WC stars are not observed (cf. references in Langer, 1987; Doom, 1987). Evolutionary calculations without overshooting avoid both discrepancies. We conclude that convective overshooting is not very efficient in massive H-burning stars, but that the Schwarzschild-criterion may be a fair approximation in order to determine the size of the convective core.

2) To predict the spatial extent of ICZs is especially complicated, since they establish in regions of varying mean molecular weight, and the problem of semiconvection is encountered (cf. Langer et al., 1983; 1985). Langer (1987) argued, that in order for massive stars to terminate their nuclear evolution as a WR star of type WNL, the mass of the ICZ is required to exceed a critical value of $M_{I C Z} \geq \dot{M}_{W R} \cdot \tau_{H e} \simeq 10-20 M_{\odot}$. There are several arguments in favour of massive stars exploding as WNL stars: As mentioned above, very massive stars probably do not evolve into WNE or WC stars. Furthermore, there exist supernovae ( $\mathrm{SNe}$ )/SN remnants (SNRs) possibly originating from a WNL precursor, the most well known example being Cas A (cf. El Eid and Langer, 1986; Fesen et al., 1987). But also the SNe 1961v (Utrobin, 1984) and 1986j (Rupen et al., 1987) are suspected to originate from very massive precursors which still contain hydrogen in their outer layers, i.e. from WNL stars. Therefore we conclude, that the mass of the ICZ in very massive stars exceeds $10 M_{\odot}$.

We note that hydrogen shell burning and consequently an ICZ in very massive stars develop only if convective overshooting during central H-burning is small or negligible. This is an additional argument supporting our conclusion of point 1 ).

3) Convective core overshooting during central He-burning should be much less efficient compared to that in the H-burning phase, since mostly during central He-burning the convective core is 
growing with time, which consequently leads to the formation of a huge molecular weight barrier on top of it.

An enlarged He-burning convective core has two consequences for the WR stages of massive stars:

- It leads to more extreme surface compositions in WC stars, i.e. to smaller surface helium concentrations but higher $\mathrm{C}$ - and $\mathrm{O}$-abundances. The surface helium mass fraction $Y$ can be roughly estimated to be larger than a certain value, depending on the mass of the convective core $M_{c c}: Y \geq\left(M_{W C}-M_{c c}\right) /\left(\dot{M}_{W C} \cdot \tau_{H e}\right)$, where $M_{W C}$ is the mass of the WC star, $\dot{M}_{W C}$ its average mass loss rate, and $\tau_{H e}$ its He-burning lifetime.

- It leads to a larger upper limit for the mass of WC stars. Such limit exists since WR stars of higher mass have larger envelope masses $M_{e n v}=M_{W R}-M_{c c}$ and shorter He-burning lifetimes, but presumably no larger mass loss rates. The mass limit is determined by $M_{e n v}\left(M_{W R}\right)=$ $M_{W R}-M_{c c} \geq \dot{M}_{W R} \cdot \tau_{H e}$.

Concerning the first point, observations are not yet sufficiently accurate in order to draw conclusions (cf. Torres, 1987). For the second point, we can conclude at least that no overshooting is consistent with observations, since it leads to a maximum mass for WC stars of $\sim 60 M_{\odot}$, which is consistent with the absence of very massive WC stars mentioned above ( $\mathrm{cf}$. also: Langer and Kiriakidis, 1988).

\section{Summary}

- The convective core size in very massive H-burning stars may well be approximated by the Schwarzschild criterion.

- In sufficiently massive stars the extension of the intermediate convection zone exceeds $10-20 M_{\odot}$.

- The convective core size in massive He-burning stars can hardly be restricted by observations. However, the absence of very massive WC stars is consistent with the case of no overshooting at all.

Theoretical evolutionary sequences taking into account the above points lead to the following scheme, which is basically consistent with recent papers of Schild and Maeder (1984), Langer (1987), and Doom (1987):

most massive stars $\rightarrow$ WNL $\rightarrow$ SN

very massive stars $\rightarrow \mathrm{WNL} \rightarrow \mathrm{WCE} \rightarrow \mathrm{SN}$

massive stars $\rightarrow \mathrm{WNE} \rightarrow \mathrm{WCL} \rightarrow \mathrm{SN}$

less massive stars $\rightarrow$ WNE $\rightarrow \mathrm{SN}$.

The author gratefully acknowledges a travel grant of the Deutsche Forschungsgemeinschaft.

\section{References}

Doom, C.: 1987, Astron. Astrophys. Letters 182, L43

El Eid, M.F., Langer, N.: Astron. Astrophys. 167, 274

Fesen, R.A., Becker, R.H., Blair, W.P.: 1987, Astrophys. J. 313, 378

Langer, N.: 1987, Astron. Astrophys. Letters 171, L1

Langer, N., Sugimoto, D., Fricke, K.J.: 1983, Astron. Astrophys. 126, 207

Langer, N., El Eid, M.F., Fricke, K.J.: 1985, Astron. Astrophys. 145, 179

Langer, N., El Eid, M.F.: 1986, Astron. Astrophys. 167, 265

Langer, N., Kiriakidis, M.: 1988, this volume

Mermilliod, J.C., Maeder, A.: 1986, Astron. Astrophys. 158, 45

Prantzos, N., Doom, C., Arnould, M., de Loore, C.: 1986, Astrophys. J. 304, 695

Rupen, M.J., v. Gorkom, J.H., Knapp, G.R., Gunn, J.E., Schneider, D.P.: 1987, Astron. J. 94, 61

Schild, H., Maeder, A.: 1984, Astron. Astrophys. 136, 237

Torres, A.V.: 1987, Astrophys. J., in press

Utrobin, V.P.: 1984, Astrophys. Space Sci. 98, 115 